\title{
Relationship of obesity with B vitamin status: analysis of NDNS data from UK women of reproductive age
}

\section{Abstract}

Pre-pregnancy obesity is associated with a 2-fold increased risk of Neural Tube Defects (NTD), an effect not explained by lower dietary folate intake, or non-use of folic acid supplements (as recommended globally to women before and in early pregnancy for NTD prevention). While the exact mechanism linking NTD and obesity is poorly understood, it is possible that a compromised status or metabolism of folate and/or of the closely related micronutrients (vitamin B12, B6 and riboflavin) may be involved. To date however, this hypothesis has not been adequately explored. This study therefore aimed to investigate the relationship of obesity with folate and related B vitamin biomarkers in a representative cohort of non-pregnant UK women of reproductive age. Data were accessed from the most recent UK National Diet and Nutrition Survey (NDNS; Years 7-8; 2015-16), a rolling cross-sectional survey designed to gather information from a representative sample of the UK population on nutrient intakes, food consumption and nutritional status. Data for women aged 16-45 years (non B vitamin supplement users; n 364), were extracted on: anthropometry (height, weight, body mass index [BMI] and waist circumference [WC]), dietary intakes of B vitamins and biomarkers for serum and red blood cell folate, vitamin B12 (total serum B12 and holotranscobalamin), vitamin B6 (plasma pyridoxal-5-phosphate [PLP]) and riboflavin (erythrocyte glutathione reductase activation coefficient EGRAac]). Prevalence of overweight and obesity (i.e. BMI: $25-30 \mathrm{~kg} / \mathrm{m}^{2}$ and $>30 \mathrm{~kg} / \mathrm{m}^{2}$ ) was $28 \%$ and $22 \%$ respectively, whilst abdominal obesity (i.e. WC $>88 \mathrm{~cm}$ ) was present in $31 \%$ of the women. Total serum B12 (r $-0.215, P$ $0.012)$ and PLP (r -0.270, P 0.002) were negatively correlated with WC, with similar, albeit weaker, correlations found for BMI. Correspondingly, women with abdominal obesity compared to those without, had a lower status of total serum B12 (Median values of: $212 \mathrm{v} 249 \mathrm{v} \mathrm{pmol} / \mathrm{L} ; P$ 0.049), B6 (31.5 v $40.5 \mathrm{nmol} / \mathrm{L} ; P$ 0.002) and EGRac $(1.43 \mathrm{v} 1.36 ; \mathrm{P} 0.031)$. No differences in B vitamin dietary intake were observed by categories of abdominal obesity. Abdominal obesity may be a risk factor for low status of both vitamins B12 and B6, independently of dietary intake. Further investigation to elucidate the potential underlying mechanism is warranted. These findings also highlight the importance of taking abdominal obesity into account, in addition to BMI, when examining possible adverse impacts of obesity on micronutrient status.

\section{Conflict of Interest}

There is no conflict of interest 